\title{
CONSTRUÇÃo E VALIDAÇÃO dE INSTRUMENTOS DE COLETA DE DADOS PARA O PERÍOdo PERIOPERATÓrio de cirurgia Cardíaca
}

Luzia Elaine Galdeano ${ }^{1}$

Lidia Aparecida Rossi

Galdeano LE, Rossi LA. Construção e validação de instrumentos de coleta de dados para o período perioperatório de cirurgia cardíaca. Rev Latino-am Enfermagem 2002 novembro-dezembro: 10(6):800-4.

Este estudo teve como objetivo construir e validar instrumentos de coleta de dados para a identificação de diagnósticos de enfermagem de pacientes adultos que se encontram no período perioperatório de cirurgia cardíaca. Os instrumentos de coleta de dados propostos foram fundamentados no modelo conceitual de Wanda Horta. Para construção dos instrumentos foi realizado um levantamento bibliográfico com o objetivo de levantar sinais e sintomas importantes, que caracterizariam os diagnósticos de enfermagem. Para a validação de aparência e conteúdo dos instrumentos de coleta de dados, elaboramos um questionário, que foi entregue a cinco enfermeiros convidados para realizar a validação. Todos os profissionais solicitados para realizar a validação expressaram que os instrumentos favorecem a formulação dos diagnósticos de enfermagem e que os dados ou questões são suficientes para identificar alterações nas necessidades básicas.

DECRITORES: coleta de dados, cirurgia cardíaca, diagnóstico de enfermagem

\section{ELABORATION AND VALIDATION OF DATA COLLECTION INSTRUMENTS FOR THE PERIOPERATIVE PERIOD OF CARDIAC SURGERY}

The purpose of this study was to elaborate and validate data collection instruments in order to identify nursing diagnosis in adult patients in the perioperative period of a cardiac surgery. The data collection instruments proposed were based on Wanda Horta's conceptual model. The elaboration of the instruments was based on a literature review with the aim to find important signs and symptoms that could characterize nursing diagnosis. For the validation of the data collection instruments, authors elaborated a survey that was validated by five nurses. All the professionals who were asked to validate it answered that the instruments helped in the identification of the nursing diagnosis and that the data or questions were enough to identify changes in basic needs.

DESCRIPTORS: data collection, nursing diagnosis, cardiac surgery

\section{CONSTRUCCIÓN Y VALIDACIÓN DE INSTRUMENTOS DE RECOLECCIÓN de datos para el período perioperatório de cirugía cardiaca}

Este estudio tiene como objetivo construir y validar instrumentos de recolección de datos para la identificación de diagnósticos de enfermería en pacientes adultos que se encuentran en el período perioperatório de cirugía cardiaca. Los instrumentos de recolección propuestos fueron fundamentados en el modelo conceptual de Wanda Horta. Para la construcción de los instrumentos se realizó una búsqueda bibliográfica con el objetivo de identificar los signos y síntomas importantes que caracterizan los diagnósticos de enfermería. Para la validación de la apariencia y el contenido de los instrumentos, elaboramos un cuestionario que fue entregado a cinco enfermeros invitados para este propósito. Todos los profesionales que realizaron esta función, expresaron que la estructura de los instrumentos favorecen la formulación de los diagnósticos de enfermería y que los datos o preguntas son suficientes para identificar alteraciones en las necesidades básicas del paciente.

DESCRIPTORES: recolección de datos, cirugía cardiaca, diagnóstico de enfermería

\footnotetext{
${ }^{1}$ Aluna do Programa de Pós-Graduação, Área Fundamental, nível mestrado, e-mail: egaldean@eerp.usp.br; ${ }^{2}$ Professor Doutor, e-mail: rizzardo@eerp.usp.br. Escola de Enfermagem de Ribeirão Preto da Universidade de São Paulo, Centro Colaborador da OMS para o desenvolvimento da pesquisa em enfermagem
} 


\section{INTRODUÇÃO}

$\boldsymbol{O}$ avanço da cirurgia cardíaca gerou um maior desenvolvimento e expansão dos cuidados de enfermagem a pacientes com doenças cardiovasculares. Os cuidados de enfermagem passaram a ser fundamentais para a recuperação do paciente submetido a uma cirurgia cardíaca $^{(1)}$. Nesse contexto, a enfermagem vem aprimorando seus conhecimentos e propondo novas alternativas de assistência ${ }^{(2)}$, desenvolvendo uma metodologia própria de trabalho, fundamentada em um método científico, isto é, fundamentada no processo de enfermagem.

O processo de enfermagem tem sido considerado uma possibilidade de resposta a sérios questionamentos referentes à qualidade do cuidado e desenvolvimento científico da enfermagem ${ }^{(3)}$.

O processo de enfermagem é definido como a "dinâmica das ações sistematizadas e inter-relacionadas visando o ser humano. É caracterizado pelo interrelacionamento e dinamismo de suas fases ou passos"(4). $\mathrm{Na}$ sua forma atual, o processo de enfermagem encontrase descrito em cinco fases seqüenciais e interrelacionadas: coleta de dados, diagnóstico de enfermagem, planejamento, implementação e avaliação ${ }^{(5)}$.

"O levantamento de dados é o alicerce no qual se fundamenta o cuidado de enfermagem" ${ }^{\text {(3) }}$. O histórico de enfermagem (coleta de dados) é a primeira etapa do processo de enfermagem, definida como: "um roteiro sistematizado para o levantamento de dados do ser humano (significativos para o enfermeiro) que tornam possível a identificação de seus problemas"(4).

No período perioperatório, a utilização de instrumentos de coleta de dados permite a identificação das necessidades humanas básicas do paciente e estabelece um fluxo de comunicação entre a unidade de internação cirúrgica, centro cirúrgico e recuperação anestésica, garantindo a continuidade da assistência de enfermagem ao paciente cirúrgico, nos períodos pré, trans e pós-operatório ${ }^{(6)}$

A utilização de um instrumento de coleta de dados permite a identificação de um maior número de problemas, quando comparado à anotação de enfermagem ${ }^{(7)}$. Em um estudo, concluiu-se que a observação sistematizada, mediante a utilização de um instrumento aplicado a paciente permitiu a identificação de 2184 problemas em 46 pacientes, enquanto a anotação de enfermagem rotineira em prontuários permitiu a identificação de somente 662 problemas $^{(7)}$.

Alguns autores propõem a utilização das características definidoras e dos fatores relacionados, da taxonomia da NANDA (North American Nursing Diagnosis Association), para delimitar o conteúdo de um instrumento de coleta de dados para a identificação dos diagnósticos de enfermagem ${ }^{(3)}$.

Encontramos um estudo em que a autora validou um instrumento, elaborado pelo Grupo de Estudos em Sistematização da Assistência de Enfermagem de um hospital, para a utilização junto a pacientes cardiopatas, internados na unidade de cardiologia. O objetivo da autora foi tornar o instrumento mais específico, de forma a favorecer a enfermagem em sua prática profissional, e subsidiar o ensino e a pesquisa em enfermagem, referente ao cuidado ao paciente cardiopata ${ }^{(8)}$.

Na revisão de literatura não foram encontrados estudos que propõem instrumentos de coleta de dados para os três períodos operatórios (pré, trans e pósoperatórios). Encontramos um estudo em que foi realizada a validação de um instrumento de coleta de dados para a identificação de diagnósticos de enfermagem de pacientes cardíacos no período pré-operatório ${ }^{(2)}$.

Para que a assistência de enfermagem perioperatória seja individualizada, contínua e planejada é necessário compreender o paciente em todas as fases, tornando fundamental a avaliação pré-operatória, transoperatória e pós-operatória de enfermagem ${ }^{(9)}$.

Sabemos que as necessidades básicas afetadas do paciente, nos períodos pré e trans-operatórios interferem diretamente nas suas necessidades no período pós-operatório. Um paciente que apresenta dúvidas quanto à cirurgia e ao tipo de anestesia a que será submetido, poderá apresentar um alto nível de ansiedade, interferindo na sua recuperação pós-operatória ${ }^{(1)}$.

Diante desse contexto e motivadas pelo desejo de implantar uma avaliação de enfermagem perioperatória sistematizada, com o objetivo de promover a continuidade da assistência de enfermagem, decidimos construir três instrumentos de coleta de dados: um para o período préoperatório, visando conhecer o paciente, suas necessidades, estabelecer uma relação enfermeiropaciente, além de identificar o potencial de complicações; um para o período trans-operatório, com o objetivo de identificar intercorrências ou complicações que possam interferir no pós-operatório; e outro para o período pós- 
operatório, com o objetivo de identificar fatores de risco, sinais e sintomas que caracterizem problemas de enfermagem.

Acreditamos que os diagnósticos de enfermagem encontrados servirão de base para a elaboração do plano de cuidados de enfermagem, considerando a individualidade do paciente. Sendo assim, o objetivo desse estudo é construir e validar instrumentos de coleta de dados para a identificação de diagnósticos de enfermagem de pacientes adultos, no período perioperatório de cirurgia cardíaca.

\section{METODOLOGIA}

A construção dos instrumentos de coleta propostos

Os instrumentos de coleta de dados propostos foram fundamentados no modelo conceitual de Wanda Horta $^{(4)}$, que se baseia nas necessidades humanas básicas propostas por Maslow ${ }^{(10)}$ e na classificação de Mohana $^{(11)}$, com o objetivo de identificar os diagnósticos de enfermagem no período perioperatório de cirurgia cardíaca. Optamos pelo modelo de Horta ${ }^{(4)}$ por considerálo um modelo que permite a avaliação do paciente como um todo indivisível, com seus componentes bio-psicosócio-espirituais.

Para a construção dos instrumentos foi realizado um levantamento bibliográfico para cada necessidade básica, com o objetivo de levantar sinais e sintomas importantes, que caracterizem diagnósticos de enfermagem de pacientes que se encontram no período perioperatório de cirurgia cardíaca. Procuramos agrupar o máximo possível de elementos que possam caracterizar os diagnósticos de enfermagem, tendo por base a literatura e a nossa experiência enquanto enfermeiras em uma unidade de recuperação anestésica.

Validação de aparência e conteúdo

A validação consiste em avaliar se o instrumento mede aquilo que se propõe a medir ${ }^{(12)}$. Para a validação de aparência e conteúdo dos instrumentos de coleta de dados, elaboramos um questionário, que foi entregue a cada enfermeiro convidado para realizar a validação. Convidamos enfermeiras (quatro docentes e uma doutoranda da Escola de Enfermagem de Ribeirão Preto-
USP), com experiência em cardiologia e conhecimento em diagnóstico de enfermagem, no intuito de identificar se os itens dos instrumentos representariam o universo do conteúdo e se permitiriam alcançar o objetivo traçado ${ }^{(2)}$. O questionário elaborado para a validação compreende cinco questões norteadoras, relacionadas à avaliação dos enfermeiros quanto à forma de apresentação e conteúdo dos instrumentos, e sugestões quanto à retirada, acréscimo ou modificações dos itens.

Os resultados ora apresentados compreendem parte de um estudo intitulado Diagnósticos de enfermagem de pacientes no período perioperatório de cirurgia cardíaca, aprovado pelo Comitê de Ética e Pesquisa do Hospital das Clínicas da Faculdade de Medicina de Ribeirão Preto.

\section{RESULTADOS}

O instrumento elaborado para o levantamento de dados no período pré-operatório recaiu sob a forma de um questionário, constituído de perguntas aberto-fechadas (que permitem aos respondentes utilizar suas próprias palavras em suas respostas) e check-list (que permite o direcionamento e o registro das observações do enfermeiro) $)^{(12)}$.

Os instrumentos para os períodos, trans e pósoperatório imediato são do tipo check-list. Optou-se por esse formato para servir de guia de orientação para a enfermagem, assegurando que não sejam omitidos dados considerados essenciais.

Nesses períodos, para cada necessidade básica, os dados foram separados por hora ( $1^{\mathrm{a}}$ hora, $2^{\mathrm{a}}$ hora, até a $6^{a}$ hora), com o objetivo de facilitar o registro e a visualização dos dados. No trans-operatório, a $1^{\text {a }}$ hora corresponde ao início da cirurgia e, no pós-operatório, corresponde à hora de chegada do paciente na recuperação pós-cirurgia cardíaca.

Ao final de cada item dos instrumentos, elaboramos um espaço intitulado outras considerações importantes destinado ao relato de impressões do enfermeiro ou outros registros que se fizerem necessários. Assim, as necessidades humanas que não constituem um item do instrumento, como necessidades de regulação hormonal, de ambiente, de amor, de liberdade, de recreação, de lazer, entre outras, poderão ser registradas nesse espaço. 
Como já afirmado anteriormente, o instrumento de coleta de dados para o período trans-operatório foi separado por hora, da $1^{\text {a }}$ à $6^{\mathrm{a}}$ hora, porém a cirurgia pode durar mais de seis horas, nesse caso, os dados referentes à $7^{a}$ hora em diante deverão ser registrados no espaço outras considerações importantes.

Ressaltamos que algumas necessidades básicas foram agrupadas devido à grande inter-relação existente entre elas, de forma a facilitar a coleta de dados. Desse modo, as necessidades de exercício e atividade física, de mecânica corporal, de motilidade e de locomoção foram agrupadas em um só item: necessidade de locomoção e movimentação. Ainda um outro agrupamento foi o da necessidade de regulação neurológica com o de percepção, constituindo somente um item: o de necessidade de senso-percepção.

Todos os profissionais solicitados para realizarem a validação expressaram que os instrumentos favorecem a formulação dos diagnósticos de enfermagem e que os dados ou questões são suficientes para identificar alterações nas necessidades básicas. Todos também expressaram que não existe repetição de dados ou questões nas diferentes necessidades.

Foram sugeridas algumas alterações, em sua maioria, relacionadas à forma de apresentação dos instrumentos. As sugestões foram aceitas, aumentando a clareza dos itens e facilitando a leitura e compreensão dos instrumentos.

Uma das alterações foi a substituição de raça por cor, no item referente à identificação no período préoperatório. A necessidade dessa substituição foi justificada pela intensa mistura de raças existente em nosso país, tornando difícil a sua classificação. Uma outra sugestão foi o acréscimo da investigação da dor torácica em repouso ou aos esforços, ajudando na identificação da possível causa da dor.

A necessidade de circulação foi substituída por necessidade de regulação vascular, conforme

\section{REFERÊNCIAS BIBLIOGRÁFICAS}

1 - Keller SM. Nursing management of the cardiac surgical patient. In: Dolan JT. Critical care nursing: clinical management through the nursing process. Philadelphia: F.A Davis; 1991. p.957-83.

2 - Riccio GMGEt, Sampaio LABN, Faria MdeFG, Caracciolo LT, Ribeiro FG, Cruz DMda. Validação de instrumento de levantamento de dados para formulação de diagnósticos de enfermagem. Rev Soc Cardiol Estado de São Paulo 1995 maio/jun.; 5(3, Supl A):1-16. orientação de um dos profissionais, por tratar-se de uma terminologia mais adequada, considerando o referencial teórico adotado.

As questões referentes às necessidades de ensino-aprendizagem, segurança e auto-estima, no período pré-operatório, foram modificadas conforme sugestão de três profissionais. Foi sugerida a utilização de linguagem mais simples, de forma a melhorar o entendimento do paciente quanto a essas questões. Desse modo, foi necessária a reformulação das perguntas e a aplicação dos instrumentos, na prática, a um número significativo de pacientes ${ }^{(2)}$.

\section{CONSIDERAÇÕES FINAIS}

Considerando os objetivos deste estudo, os resultados obtidos favorecem um direcionamento da assistência com base no processo de enfermagem, subsidiam estudos similares e fornecem instrumentos de coleta de dados válidos e confiáveis, com o objetivo de identificar os diagnósticos de enfermagem no período perioperatório de cirurgia cardíaca. Os instrumentos construídos poderão ser reproduzidos para a identificação dos diagnósticos de enfermagem em outras áreas.

Consideramos fundamental o desenvolvimento e a divulgação de estudos relacionados a esse assunto, no intuito de direcionar o raciocínio dentro de uma base de conhecimento, permitindo uma análise em profundidade dos problemas do paciente que demandam ações específicas de enfermagem, contribuindo para o desenvolvimento científico da profissão ${ }^{(2)}$.

Acreditamos que o conhecimento dos problemas de saúde de um grupo de pacientes com características comuns, irá direcionar a assistência de enfermagem, fornecendo subsídios para a elaboração do plano de cuidados, implementação de intervenções, treinamento e qualificação da equipe ${ }^{(13)}$.

3 - Pimenta Cade M, Cruz DALM da, Graziano KU, Kimura M, Miyadahira AMK. O ensino da avaliação do paciente: delineamento do conteúdo pelo diagnóstico de enfermagem. Rev Latino-Am Enfermagem 1993 julho; 2(1):69-76.

4 - Horta WdeA. Processo de enfermagem. São Paulo: EDUSP; 1979.

5 - Carvalho EC, Rossi LA. Modelos, estruturas e teorias de enfermagem: aplicação através do processo de enfermagem. In: Garcia TR, Pagliuca LMF, coordenadores. A construção do conhecimento em enfermagem: coletânea de trabalhos. Fortaleza: RENE; 1998. 
6 - Oliveira HF, Aquino VLA de, Calbo VC. A percepção da enfermeira sobre o sistema da assistência de enfermagem perioperatória. Rev Paul Enfermagem 1990 set/dez.; 9(3):97101.

7 - Horta WdeA. A observação sistematizada na identificação dos problemas de enfermagem em seus aspectos físicos. Rev Bras Enfermagem 1974; 27(2):214-9.

8 - Michel JLM. Validação de instrumento para coleta de dados de pacientes pacientes coronariopatas. [Dissertação]. São Paulo: Escola Paulista de Medicina/UFSP; 1999.

9 - Castellanos BEP, Jouclas VMG. Assistência de enfermagem perioperatória: um modelo conceitual. Rev.Esc Enfermagem USP 1990 dezembro; 24(3):359-70.

10 - Maslow AH. Motivation and personality. 2. ed. New York: Harper \& Row; 1970.

11 - Mohana J. O mundo e eu. 2. ed. Rio de Janeiro: Agir; 1964.

12 - Polit D, Hungler BP. Fundamentos de pesquisa em enfermagem. Porto Alegre: Artes Médicas; 1995

13 - Cruz D.de ALM. Os diagnósticos de enfermagem no ensino e na pesquisa. Rev Esc Enfermagem USP 1992 dezembro; 26(3):427-34. 